\title{
The close-limit approximation to Neutron Star Collisions
}

\author{
Gabrielle D. Allen ${ }^{(1)}$, Nils Andersson ${ }^{(2)}$, Kostas D. Kokkotas ${ }^{(3)}$, \\ Pablo Laguna ${ }^{(4)}$, Jorge A. Pullin ${ }^{(4)}$ and Johannes Ruoff ${ }^{(5)}$ \\ (1) Max Planck Institute for Gravitational Physics \\ The Albert Einstein Institute, D-14473 Potsdam, Germany \\ (2) Department of Mathematics \\ University of Southampton, Southampton SO17 1BJ, UK \\ (3) Department of Physics \\ Aristotle University of Thessaloniki, Thessaloniki 54006, Greece \\ (4) Center for Gravitational Physics \& Geometry \\ Penn State University, University Park, PA 16802, USA \\ (5) Institut für Astronomie und Astrophysik \\ Universität Tübingen, D-72076 Tübingen, Germany
}

\begin{abstract}
We develop a close-limit approximation to the head-on collision of two neutron stars similar to that used to treat the merger of black hole binaries. This approximation can serve as a useful benchmark test for future fully nonlinear studies. For neutron star binaries, the close-limit approximation involves assuming that the merged object can be approximated as a perturbed, stable neutron star during the ring-down phase of the coalescence. We introduce a prescription for the construction of initial data sets, discuss the physical plausibility of the various assumptions involved, and briefly investigate the character of the gravitational radiation produced during the merger. The numerical results show that several of the merged objects fluid pulsation modes are excited to a significant level.
\end{abstract}

04.30.Nk, 04.40.Nr, 04.25.Dm

\section{INTRODUCTION}

The inspiral and subsequent merger of compact binaries is one of the most promising sources of gravitational radiation for the new generation of interferometric detectors (LIGO, VIRGO, GEO600, TAMA). Once these instruments come online, we hope to learn much about physics in strong gravitational fields and at super-nuclear densities. This is an exciting prospect, but we need reliable theoretical models of the relevant gravitational-wave signals to dig them out of what is likely to be a significantly noisy data stream. Hence, a huge effort is presently being made to model the late stages of binary inspiral, both for black holes and neutron stars. For the inspiral phase the post-Newtonian approximation scheme has been pushed to very high orders [1], and the available results are now at a rather satisfactory level of accuracy. For the final merger of the two binary companions, our understanding is not yet at a comparable level. To completely model the collision of two compact objects, one must resort to numerical relativity and fully nonlinear simulations assuming no symmetries. This provides a great computational challenge, and truly reliable results may not be available for several years.

In the last few years, our understanding of black hole collisions has improved considerably. An important reason for the recent advances is the parallel development of the numerical approach and approximate methods. In fact, the so-called close-limit approximation (in which the late stages of the merger is modeled by considering the two black holes as a single perturbed one) is in remarkable agreement with the numerical simulations [2,3]. We thus have a powerful benchmark test for the fully numerical schemes, which gives us some confidence in the physical picture that emerges. Of course, a considerable amount of work remains before the black-hole problem is studied in complete generality. Most importantly, the calculations must be generalized to include rotational effects 伍.

Compared to the collision of two black holes, the merging of two neutron stars involves, in addition to geometrodynamics, a non-trivial amount of "dirty" physics associated with the stellar fluid and a strong magnetic field . In the initial stages of collision, a shock wave will be generated; the merged object will heat up dramatically; the electromagnetic fields of the two stars will become intertwined; and a strong burst of energy may result. Even though the details remain to be understood, the merger is believed to lead to (most of) the observed cosmological gamma-ray bursts [5]. From a gravitational point of view, this is interesting since it means that gamma ray bursts should be accompanied by a burst of gravitational radiation. Thus, there is a possibility that future gravitational-wave observations will help shed light on the gamma-ray puzzle. Anyway, at the later stages of the merger, several avenues are open. If the merged object is too massive to form a neutron star, it will collapse to form a black hole. But for an interesting range of masses the collapse may be temporarily halted. A supermassive neutron star can be supported either by thermal 
pressure or by rotation [6,7]. In these cases, the final collapse will occur only after the object either cools or spins down. The likely extreme rotation of the merged object is relevant also for other reasons. A rapidly rotating stellar object can be both dynamically and secularly unstable. In the first case, the star spins so fast that matter is ejected from the equator. Via the so-called bar-mode instability, the core will shed some of its mass and a sufficient amount of angular momentum for it to become dynamically stable [8]. Once sufficient angular momentum has been shed to bring the rotation rate of the merged object below the dynamical Kepler limit, secular instabilities may come into play [9]. Based on recent results, we would expect the instability associated with the merged objects $r$-modes to spin the core down to a period longer than $10 \mathrm{~ms}$ in a year or so [10 13]. The merged object should, in fact, evolve almost exactly like a newly born neutron star (up to the eventual collapse in the case of supermassive configurations). Hence, the formation of a massive neutron star via merger of two less massive ones could be followed by a detectable [14.15] gravitational wave signal due to the $r$-modes.

Clearly, it is a very difficult task to model all the aspects of the merger in general relativity. On the other hand, the payoff from detecting and understanding these events is enormous. Neutron star mergers provide unique laboratories for physics at an extreme level. Given an understanding of the single pieces of the merger puzzle, we can hope to put together an accurate picture of the entire event. This paper presents an investigation into the late stages of binary neutron star mergers. Inspired by the success of the close-limit approximation for colliding black holes, we develop an analogous framework for the case of neutron stars. Although the close-limit approach to compact object mergers is unlikely to be as successful in the case of neutron stars (see later discussion), our hope is to gain insight into the character of the gravitational waves that emerge from these events. Furthermore, the framework that we develop should serve as a useful benchmark test for future fully general relativistic hydrodynamical simulations.

In the close-limit approach, the late stages of binary merger are studied by assuming that the initial configuration can be viewed as (in some sense small) perturbations of the final object. The background is either a known analytic or a simple numerical solution. For black hole binaries, the background is a Schwarzschild or Kerr spacetime, depending on whether the remnant is a slowly or a rapidly rotating black hole. For the situation considered in the present paper, head-on neutron star mergers, the background can be either a black hole or a non-rotating relativistic star. The approximation that we will develop is only relevant in the latter case. The case when a black hole is formed requires a study of gravitational collapse, and is beyond our present aims. One may argue that the collision of two neutron stars should typically lead to an object that is too massive to support itself against gravity, and which must therefore collapse to a black hole. If that were the case, we would be restricted to the presumably small subset of mergers that involve less massive neutron stars. However, as mentioned above, supermassive neutron stars can (at least temporarily) be supported by both thermal pressure and rotation. Since the merged object is likely to heat up to temperatures beyond $10^{11} \mathrm{~K}$ and ought to spin close to the Kepler limit due to conservation of angular momentum, these effect will be highly relevant. If the object is too massive to form a cold non-spinning neutron star, collapse will inevitably follow either on the cooling timescale (of the order of $10 \mathrm{~s}$ for cooling due to neutrino emission) or the spin-down timescale (which depends entirely on the mechanism due to which the merged object spins down). Since both of these time-scales are orders of magnitude longer than the dynamical timescale of the merged object (typically at the ms level) the immediate aftermath of merger ought to be attainable within our close-limit approach.

In the case of colliding black holes, the recent studies show that the emerging gravitational waves are dominated by the quasinormal modes of the final black hole. Intuitively, we expect a similar result for merging neutron stars. The merged object will pulsate wildly. This then leads to a characteristic gravitational-wave signal containing several of the nonradial modes of oscillation of the newly formed star. Provided that these modes can be identified in the gravitational-wave data, their particulars can be used to put strong constraints on the mass and the radius (as well as the equation of state) of the star 16 18]. This is an interesting idea, but it requires that the modes are excited to detectable levels. Previous simulations indicate only that the fluid $f$-modes and $p$-modes, as well as the gravitationalwave $w$-modes are excited when a non-rotating star is perturbed generically [19,20]. With the present approximation, we can estimate the actual level of excitation of these modes at the late stages of neutron star merger. This will provide information that can be used to assess the detectability of these events.

Time evolutions of black hole and neutron star perturbations have recently received considerable attention. For non-rotating black holes, the dynamics of perturbations can be investigated via the Zerilli equation [21]. Studies of such problems have led to a much improved understanding of the dynamics of non-rotating black holes. A similar approach has also been used for perturbed rotating black holes. Rotating black-hole perturbations have been studied in the time-domain by solving the Teukolsky equation [22]. For perturbations of non-rotating neutron stars, there are many possible formulations, even within a particular choice of gauge. The results we present for the evolution of initial data representing neutron star head-on mergers were obtained using two independent codes based on perturbative variables within the Regge-Wheeler gauge [19,20].

Given a reliable numerical code to evolve perturbations of a single neutron star, the close-limit calculation only requires the construction of relevant initial data. An obvious fundamental difference between close-limit initial data sets for black hole collisions and those involving neutron stars is the presence of a horizon. In fact, the success of 
the close-limit approach for black holes may to a large extent rest on the presence of the horizon. The reason is the following: The initial data for two colliding black holes corresponds to rather large perturbations only in the region inside the peak (roughly at $r=3 M$ ) of the curvature potential. In effect, the perturbative scheme is unlikely to be reliable in this region. However, the bulk of these perturbations never escape to infinity. They are scattered by the effective potential and subsequently swallowed by the final black hole. Thus, the anticipated errors in the scheme have a minute effect on the gravitational radiation that reaches the far zone. Unfortunately, the neutron star case is not masked in this way. Since one would typically only include dissipation due to gravitational radiation in the simplest models, all "errors" in our initial data must escape through this channel in the absence of a central horizon. Consequently, the outcome of close-limit neutron star collisions are likely to be rather sensitive to the particular structure of the initial data. It should also be pointed out that, the fundamental qualitative difference between the two problems makes a direct comparison between the black hole case and that of neutron stars rather dubious.

However, a more careful study of the black hole results suggests that the close-limit approach for neutron stars may not be a lost cause after all. In close-limit black-hole collisions, most of the energy radiated, if not all, comes from quadrupole $(l=2)$ quasi-normal mode $(\mathrm{QNM})$ ringing. The only aspect that distinguishes close-limit initial data from any other data is the fixed amplitude of the ringing. If we assume that a similar situation arises for neutron stars, we should focus our attention on the mode-ringing and ignore the initial burst of radiation. This is natural since one would expect the initial burst to be strongly dependent on the characteristics of the initial data. In the case of stars, this strategy is associated with an unfortunate sacrifice. The early parts of the gravitational-wave signal from the merger may contain the rapidly damped $w$-modes [23, 12]. In the proposed prescription, the inferred amplitude of these modes may well be unreliable. Actually, one would expect this to be the case on physical grounds. The problem of constructing "astrophysical" initial data in numerical relativity essentially boils down to specifying the amount of gravitational waves in the spacetime. In a neutron star spacetime, it would be natural for the initial gravitational waves to escape via the $w$-modes. Hence, an uncertainty in the amplitude of these modes is expected, given our poor understanding of the relevant astrophysical data. Taking the suggested attitude, we do not expect to be able to assess the astrophysical relevance of the $w$-modes. Instead, we should view the neutron star close-limit initial data (and the associated results) as a constraint on the amplitude of the longer lived fluid pulsation modes.

\section{CLOSE-LIMIT INITIAL DATA}

\section{A. Stellar Models}

A neutron star model in general relativity is obtained by solving the Tolman-Oppenheimer-Volkoff (TOV) system of equations:

$$
\begin{aligned}
\frac{d m}{d r} & =4 \pi r^{2} \rho_{0} \\
\frac{d \nu}{d r} & =\frac{e^{2 \lambda}}{r^{2}}\left(m+4 \pi r^{3} p_{0}\right) \\
\frac{d p_{0}}{d r} & =-\left(\rho_{0}+p_{0}\right) \frac{d \nu}{d r}
\end{aligned}
$$

where the spacetime metric is given by

$$
d s^{2}=-e^{2 \nu} d t^{2}+e^{2 \lambda} d r^{2}+r^{2} d \theta^{2}+r^{2} \sin ^{2} \theta d \varphi^{2} .
$$

Above, $\rho_{0}$ and $p_{0}$ are the density and pressure, respectively, and $m$ represents the mass inside radius $r$. It is related to the metric function $\lambda$ by

$$
e^{-2 \lambda} \equiv 1-\frac{2 m}{r} .
$$

All quantities are functions of the radial coordinate $r$ only. The construction of initial data for the close-limit approximation involves two steps: First, one needs to solve the TOV equations and obtain stellar models for both the colliding stars and the final configuration. The second step consists of a suitable superposition of colliding stars followed by a subtraction of the background star. This yields the perturbations which are then the focus of the evolution. With this in mind, we have denoted background quantities of the star formed by the merger by the subscript 0 and perturbations of these quantities by the subscript 1 . The TOV solutions of the colliding stars will be denoted by an asterisk $(*)$. For simplicity, we will only consider polytropic equations of state $p_{0}=K \rho_{0}{ }^{\Gamma}$, where $K$ and $\Gamma$ are the adiabatic constant and index, respectively. The adiabatic index $\Gamma$ is related to the polytropic index $n$ by $\Gamma=1+1 / n$. In the specific example provided later we use $\Gamma=2(n=1)$. 


\section{B. Initial Perturbations and Constraints}

The construction of astrophysically relevant initial data in numerical relativity is an outstanding problem not only from the mathematical point of view. In the present context, we would like the data to represent the initial configuration for the ring-down phase after a collision. Without the non-linear evolution that precedes the ringdown stage, the specification of initial data becomes a non-trivial "guess." In the 3+1 formulation [24] of Einstein's equations, initial data consist of the spatial metric $g_{i j}$, the extrinsic curvature $K_{i j}$ and the matter fields. These initial data contain too much freedom. Only four out of the twelve components in $\left(g_{i j}, K_{i j}\right)$ are fixed by the constraints. The remaining pieces, including the matter fields, are freely specifiable and single out the particular situation under consideration. Furthermore, it is usually not obvious which four components in $\left(g_{i j}, K_{i j}\right)$ should be obtained from solving the constraints.

The standard procedure to separate the freely specifiable data from that fixed by constraints in numerical relativity is York's conformal approach [25]. In addition, this method provides a natural framework for "superposing" solutions. This is a clear advantage given our present project. For instance, given data $\left(g_{i j}, K_{i j}\right)$ for individual black holes, it is possible to "add" these solutions and solve the constraints to obtain self-consistent, fully non-linear, initial data 26. A similar procedure can be applied to neutron star binaries. As mentioned in the previous section, our problem involves constructing perturbative initial data. Once the background matter and spacetime is specified, the construction of perturbative initial data consists of finding solutions that satisfy the Hamiltonian and momentum constraints to the order required by the perturbative expansion.

As in the non-linear case, close-limit initial data is completely characterized, for both black holes and neutron stars, by the freely specifiable data (data not fixed by the constraints) and boundary conditions. That is, both the free data and boundary conditions select from the class of solutions of the constraints those dealing with the superposition of colliding objects. In this paper, we focus the discussion on simplest possible case: the close-limit head-on collisions of neutron stars that are initially at rest. This means that the initial extrinsic curvature and matter current density vanish to all orders. Therefore, the momentum constraints are identically satisfied, and one is only required to solve the Hamiltonian constraint. More general classes of neutron star initial data are discussed in [27]. For vanishing extrinsic curvature, the Hamiltonian constraint reads:

$$
R=16 \pi \rho,
$$

where $R$ is the Ricci scalar constructed from $g_{i j}$ and $\rho$ is the matter density.

Following York's method, the spatial metric $g_{i j}$ and matter density $\rho$ are conformally transformed according to:

$$
\begin{aligned}
g_{i j} & =\phi^{4} \hat{g}_{i j} \\
\rho & =\phi^{-8} \hat{\rho},
\end{aligned}
$$

where hats denote conformal quantities. With the above transformation, Eq. (6) takes the form

$$
8 \hat{\Delta} \phi-\hat{R} \phi=-16 \pi \hat{\rho} \phi^{-3}
$$

where $\hat{\Delta} \equiv \widehat{\nabla}_{i} \widehat{\nabla}^{i}$ and $\widehat{\nabla}_{i}$ denotes covariant differentiation associated with the conformal metric $\hat{g}_{i j}$.

We will assume at this point that metric perturbations only enter via the conformal factor. That is,

$$
\begin{aligned}
\phi & =\phi_{0}+\phi_{1} \\
\hat{g}_{i j} & =\hat{g}_{i j}^{0} .
\end{aligned}
$$

However, it is important to realize that this is not a physically motivated choice. The reason for choosing data that is spatially conformally flat (i.e. $\hat{g}_{i j}^{1}=0$ ) is by no means a necessary condition for our procedure to work. In fact, for neutron stars the choice $\hat{g}_{i j}^{1}=0$ could lead to a suppression in the excitation of $w$-modes [19]. Still, the calculations are simplified considerably by this assumption, and it allows a better comparison with the black hole binary case since the initial data for multiple black holes is usually constructed assuming conformal flatness [28]. At one level, the availability of non-linear evolutions will remove this uncertainty since the free data can then be determined from the outcome of these evolutions. However, there will still be a corresponding choice to make in the specification of initial data for the nonlinear phase.

The conformal transformations $(7)$ and (8), together with the perturbative expansions (10) and (11) yield:

$$
\begin{aligned}
g_{i j}^{0} & =\phi_{0}^{4} \hat{g}_{i j}^{0} \\
g_{i j}^{1} & =4 \phi_{0}^{3} \phi_{1} \hat{g}_{i j}^{0} \\
\rho_{0} & =\phi_{0}^{-8} \hat{\rho}_{0} \\
\rho_{1} & =\phi_{0}^{-8} \hat{\rho}_{1}-8 \phi_{0}^{-9} \phi_{1} \hat{\rho}_{0} .
\end{aligned}
$$


With the above perturbative expansions, the Hamiltonian constraint takes the form

$$
8 \hat{\Delta} \phi_{1}-\left[\hat{R} \phi_{1}+48 \pi \hat{\rho}_{0} \phi_{0}^{-4}\right] \phi_{1}=-16 \pi \phi_{0}^{-3} \hat{\rho}_{1} .
$$

In summary, given the background $\left(\phi_{0}, \hat{g}_{i j}^{0}, \hat{\rho}_{0}\right)$, close-limit initial perturbations of head-on collisions of neutron stars initially at rest consists of only two quantities: the perturbation of the conformal factor, $\phi_{1}$, and the perturbation of the conformal matter density, $\hat{\rho}_{1}$. In the following, we view the density perturbation $\hat{\rho}_{1}$ as free data, with $\phi_{1}$ obtained by solving the linearized Hamiltonian constraint Eq. (16). Thus, the density perturbation $\hat{\rho}_{1}$ fully characterizes the collision, and we now turn to its specification.

\section{A Recipe for Close-limit Superposition of Stellar Models}

The key contribution of the present work is to provide a recipe for obtaining the density perturbation $\hat{\rho}_{1}$ from a suitable superposition of isolated neutron stars. To represent a "true" close-limit approximation, this superposition procedure must be such that the perturbations vanish as the separation between the stars vanish. In order to superpose two neutron stars and solve Eq. (16), it is convenient to perform a coordinate transformation that brings the 3-metric (4) into the isotropic (conformally flat) form:

$$
d s^{2}=\phi_{0}^{4}\left(d \hat{r}^{2}+\hat{r}^{2} d \theta^{2}+\hat{r}^{2} \sin ^{2} \theta d \varphi^{2}\right) .
$$

This is accomplished by setting the conformal factor to

$$
\phi_{0}=\left(\frac{r}{\hat{r}}\right)^{1 / 2},
$$

and transforming the radial-coordinate according to

$$
\frac{d \hat{r}}{d r}=e^{\lambda} \frac{\hat{r}}{r}
$$

With the metric given by (17), Eq. 16) can be trivially rewritten as a radial elliptic equation:

$$
\frac{1}{\hat{r}^{2}} \frac{d}{d \hat{r}}\left(\hat{r}^{2} \frac{d}{d \hat{r}} \phi_{1}\right)-\left[\frac{l(l+1)}{\hat{r}^{2}}+6 \pi \hat{\rho}_{0} \phi_{0}^{-4}\right] \phi_{1}=-2 \pi \phi_{0}^{-3} \hat{\rho}_{1},
$$

where we have used a standard decomposition in spherical harmonics:

$$
\begin{aligned}
& \hat{\rho}(\hat{r}, \theta, \varphi)=\hat{\rho}_{0}(\hat{r})+\sum_{l m} \hat{\rho}_{1}(\hat{r}) Y_{l m}(\theta, \varphi) \\
& \phi(\hat{r}, \theta, \varphi)=\phi_{0}(\hat{r})+\sum_{l m} \phi_{1}(\hat{r}) Y_{l m}(\theta, \varphi) .
\end{aligned}
$$

To proceed, we need to provide a superposition procedure from which $\hat{\rho}_{1}$ can be obtained. To do this, we make the following observation: All of the black-hole close-limit initial data sets considered so far have had not only the appropriate zero-separation limit but also the correct infinite-separation limit. That is, as the separation of the black holes increases, the initial data converges to that of two isolated holes. A similar behavior is in principle desirable for neutron stars. However, the situation for neutron stars is more complicated because there are no simple relations between mass, radius and density as for black holes. Hence, we are forced to use a somewhat ad hoc prescription. We neglect tidal deformations etcetera and use the following superposition of density profiles of isolated neutron stars:

$$
\hat{\rho}\left(\hat{r}^{i}\right)=\hat{\rho}_{*}\left(\hat{r}^{i}-\hat{\xi}^{i}\right)+\hat{\rho}_{*}\left(\hat{r}^{i}+\hat{\xi}^{i}\right)-\left[\hat{\rho}_{*}\left(\hat{r}^{i}-\hat{\xi}^{i}\right) \hat{\rho}_{*}\left(\hat{r}^{i}+\hat{\xi}^{i}\right)\right]^{1 / 2} .
$$

Here $\hat{\rho}_{*}$ represents the conformally transformed density profile of the individual colliding neutron stars displaced a distance $\hat{\xi}^{i}$ in conformal space. For simplicity we are assuming identical colliding stars. It is straightforward to verify that the superposition of densities (23) satisfies both the zero-separation and infinite-separation limits.

Assuming a small displacement (i.e. imposing the close-limit condition), we find that

$$
\hat{\rho}_{*}\left(\hat{r}^{i} \pm \hat{\xi}^{i}\right)=\hat{\rho}_{*}\left(\hat{r}^{i}\right) \pm \hat{\xi}^{j} \widehat{\nabla}_{j} \hat{\rho}_{*}\left(\hat{r}^{i}\right)+\frac{1}{2} \hat{\xi}^{j} \hat{\xi}^{k} \widehat{\nabla}_{j} \widehat{\nabla}_{k} \hat{\rho}_{*}\left(\hat{r}^{i}\right) .
$$


Thus

$$
\hat{\rho}=\hat{\rho}_{*}+\frac{1}{2}\left(\hat{\xi}^{i} \widehat{\nabla}_{i} \hat{\rho}_{*}\right)^{2}+\frac{1}{2} \hat{\xi}^{i} \hat{\xi}^{j} \widehat{\nabla}_{i} \widehat{\nabla}_{j} \hat{\rho}_{*},
$$

so the conformal density perturbation is given by:

$$
\begin{aligned}
\hat{\rho}_{1} & =\hat{\rho}-\hat{\rho}_{0} \\
& =\hat{\rho}_{*}-\hat{\rho}_{0}+\frac{1}{2}\left(\hat{\xi}^{i} \widehat{\nabla}_{i} \hat{\rho}_{*}\right)^{2}+\frac{1}{2} \hat{\xi}^{i} \hat{\xi}^{j} \widehat{\nabla}_{i} \hat{\nabla}_{j} \hat{\rho}_{*} .
\end{aligned}
$$

We choose coordinates such that the centers of the colliding stars lie on the $z$-axis, so the off-set vector $\hat{\xi}^{i}$ is given by:

$$
\hat{\xi}^{i}=\hat{\xi}\left(\cos \theta,-\frac{1}{\hat{r}} \sin \theta, 0\right) .
$$

Substitution of (27) into (26) yields

$$
\hat{\rho}_{1}(\hat{r}, \theta, \varphi)=\hat{\rho}_{*}(\hat{r})-\hat{\rho}_{0}(\hat{r})+\frac{1}{2} \hat{\xi}^{2}\left[\cos ^{2} \theta\left(\frac{d}{d \hat{r}} \hat{\rho}_{*}(\hat{r})\right)^{2}+\cos ^{2} \theta \frac{d^{2}}{d \hat{r}^{2}} \hat{\rho}_{*}(\hat{r})+\sin ^{2} \theta \frac{1}{\hat{r}} \frac{d}{d \hat{r}} \hat{\rho}_{*}(\hat{r})\right] .
$$

Making use of $\sqrt{4 \pi} Y_{00}=1$ and $\frac{4}{15} \sqrt{5 \pi} Y_{20}+\frac{1}{3}=\cos ^{2} \theta$, Eq. (28) can be rewritten as:

$$
\begin{aligned}
\hat{\rho}_{1}(\hat{r}, \theta, \varphi) & =\sum_{l m} \hat{\rho}_{1}(\hat{r}) Y_{l m}(\theta, \varphi) \\
& =\sqrt{4 \pi}\left[\hat{\rho}_{*}(\hat{r})-\hat{\rho}_{0}(\hat{r})+\frac{1}{2} \hat{\xi}^{2}\left\{\frac{d^{2}}{d \hat{r}^{2}} \hat{\rho}_{*}(\hat{r})+\left(\frac{d}{d \hat{r}} \hat{\rho}_{*}(\hat{r})\right)^{2}-\frac{4}{\hat{r}} \frac{d}{d \hat{r}} \hat{\rho}_{*}(\hat{r})\right\}\right] Y_{00}(\theta, \varphi) \\
& +\frac{2}{15} \sqrt{5 \pi} \hat{\xi}^{2}\left[\frac{d^{2}}{d \hat{r}^{2}} \hat{\rho}_{*}(\hat{r})+\left(\frac{d}{d \hat{r}} \hat{\rho}_{*}(\hat{r})\right)^{2}-\frac{1}{\hat{r}} \frac{d}{d \hat{r}} \hat{\rho}_{*}(\hat{r})\right] Y_{20}(\theta, \varphi) .
\end{aligned}
$$

From the above results, we deduce that the conformal density perturbation $\hat{\rho}_{1}$ has two contributions. One is a monopole part $(m=0, l=0)$,

$$
\hat{\rho}_{1}=\sqrt{4 \pi}\left[\hat{\rho}_{*}-\hat{\rho}_{0}+\frac{1}{2} \hat{\xi}^{2}\left\{\frac{d^{2}}{d \hat{r}^{2}} \hat{\rho}_{*}+\left(\frac{d}{d \hat{r}} \hat{\rho}_{*}\right)^{2}-\frac{4}{\hat{r}} \frac{d}{d \hat{r}} \hat{\rho}_{*}\right\}\right] .
$$

Since a monopole perturbation does not lead to gravitational radiation, we will not consider this contribution further. The second part is a quadrupole $(m=0, l=2)$ perturbation given by the last term in Eq. (30):

$$
\hat{\rho}_{1}=\frac{2}{15} \sqrt{5 \pi} \hat{\xi}^{2}\left[\frac{d^{2}}{d \hat{r}^{2}} \hat{\rho}_{*}+\left(\frac{d}{d \hat{r}} \hat{\rho}_{*}\right)^{2}-\frac{1}{\hat{r}} \frac{d}{d \hat{r}} \hat{\rho}_{*}\right] .
$$

This is the dominant radiative source which should be used in Eq. (20) to construct initial data. Notice that the $l=2$ perturbation (32) has the correct limit $\hat{\rho}_{1} \rightarrow 0$ when $\hat{\xi} \rightarrow 0$ independent of the stellar model used for $\hat{\rho}_{*}$. On the other hand, for the monopole perturbation (31) to vanish in the limit $\hat{\xi} \rightarrow 0$ we must have $\hat{\rho}_{*} \rightarrow \hat{\rho}_{0}$. We will now discuss a specific model in which this is the case.

\section{Defining "Realistic" Colliding Star Models}

As we already discussed in the introduction, the final stellar object that is formed by merger will be rather different from the initial stars. It will certainly be hotter and most likely spinning more rapidly since the angular momentum of the inspiral orbit must be conserved. In the case we consider here, that of head-on collision of two non-rotating neutron stars, we obviously need not worry about rotational effects. But we still we need to estimate the changes in the equation of state as the stellar material heats up during the merger. To do this we must speculate what the outcome of the collision may be, and specify a relation between the final background spacetime including $\hat{\rho}_{0}$ and the density distribution $\hat{\rho}_{*}$ of the individual initial stars. 
In order to have the correct zero- and infinite-separation limits, we must consider the relation between the masses of the colliding stars and the mass of the background star. A simplifying and to some extent reasonable condition is to assume that the mass lost during the collision is not significant, so the total mass is approximately conserved. Specifically, this implies that the total mass computed, in the physical space, from the background density $\rho_{0}$ and the total mass obtained from the superposition of densities $\rho_{*}$ in (23) are roughly the same. As we shall see, in the close-limit approximation this condition implies that properties of the colliding stellar models (for example, the radius of the stars) depend on the separation.

TOV solutions for polytropic equations of state are parameterized by the central density $\rho_{c}$, adiabatic constant $K$ and adiabatic index $\Gamma$. We assume that the collision does not modify the adiabatic index (and set $\Gamma=2$ for all models). This assumption is consistent with available numerical results 29]. Therefore, the models used to specify $\rho_{*}$ differ only in central density and adiabatic constant. We want these parameters to reflect changes in the equation of state as the temperature increases, in such a way that we retain the final, much hotter, star at zero separation. Our method for constructing polytropic equations of state for the initial stars (in terms of $\rho_{c}$ and $K$ ) in relation to that of the final star is inspired by the recent arguments of Shapiro [6] for head-on collision of neutron stars from rest at infinity.

The starting point is to notice that TOV solutions exhibit the following scalings:

$$
\begin{aligned}
M\left(\rho_{c}\right) & =\tilde{M}\left(\tilde{\rho}_{c}\right) K^{n / 2} \\
R\left(\rho_{c}\right) & =\tilde{R}\left(\tilde{\rho}_{c}\right) K^{n / 2} \\
\rho_{c} & =\tilde{\rho}_{c} K^{-n}
\end{aligned}
$$

with $M$ and $R$ the total mass and radius of the star. Above, tildes denote dimensionless quantities. To use these relations in the close-limit approach, we recall that we want our scheme to be valid in two limits: It should lead to the expected results both in the limit of zero and infinite separation. To achieve this we assume that the mass and radius of the colliding stars (index $*$ ) are related to the background star (index 0) by:

$$
\begin{aligned}
& M_{*}=M_{0}\left(\frac{2-\eta}{2}\right) \\
& R_{*}=R_{0}\left(\frac{2-\eta}{2}\right),
\end{aligned}
$$

where $\eta$ is a monotonic function of the separation $\xi$ in physical space and represents the "overlap" of the two stars. Quite naturally, $\eta$ ranges from 0 to 1 with $\eta(\xi \rightarrow 0)=0$ and $\eta(\xi \rightarrow \infty)=1$. This construction ensures that our approximation satisfies the desired limits. However, we have as yet no information about the function $\eta$ for intermediate separations. Such information could be obtained from fully numerical studies of merging stars. In the present study we make a natural, albeit quite arbitrary, choice. We assume that in the close-limit regime the overlap function is linear in the separation of the colliding stars and use

$$
\eta(\xi)=\frac{\xi}{R_{0}} .
$$

Substitution of (36) and (37) into (33) and (34), respectively, now yields

$$
\frac{\tilde{M}_{*}}{\tilde{M}_{0}}=\frac{\tilde{R}_{*}}{\tilde{R}_{0}}=\left(\frac{2-\eta}{2}\right)^{-1}\left(\frac{K_{0}}{K_{*}}\right)^{\frac{n}{2}} .
$$

Eq. (39) implies

$$
\frac{\tilde{M}_{*}}{\tilde{R}_{*}}=\frac{\tilde{M}_{0}}{\tilde{R}_{0}} .
$$

Since the ratio $\tilde{M} / \tilde{R}$ is a monotonic function of $\tilde{\rho}_{c}$, Eq. 40 ) yields

$$
\frac{\tilde{M}_{*}}{\tilde{M}_{0}}=\frac{\tilde{R}_{*}}{\tilde{R}_{0}}=1 .
$$

Hence, from (39), (41) and (35), one obtains the following relationships between adiabatic constants and central densities: 


$$
\begin{aligned}
& K_{*}=K_{0}\left(\frac{2-\eta}{2}\right)^{2 / n} \\
& \rho_{c}^{*}=\rho_{c}^{0}\left(\frac{2-\eta}{2}\right)^{-2} .
\end{aligned}
$$

In the limit $\eta \rightarrow 1$, these scalings reduce to the case of two polytropic stars colliding head-on from rest at infinity [6]. Here, we will use (42) and (43) to relate the polytropic equations of state for the initial and the final stars, also for intermediate separations.

It is important to point out that, if the amount of mass lost during the collision is not negligible conservation of mass is not given by $M_{0}=2 M_{*}$. As is clear from Eq. (36), conservation of mass would be given by $M_{0}=2 M_{*}$ only in the case of infinite separation. For the situation we consider, conservation of mass is demonstrated by comparing the mass $M_{0}$ computed from $\rho_{0}$ to that from $\rho$ in (23). It is the mass obtained from the superposition rule (23) that takes correctly into account the double counting in the overlap of the density profiles of the colliding stars.

\section{NUMERICAL RESULTS}

Our numerical procedure to construct initial data consists of first solving, using a fourth-order Runge-Kutta integrator, the TOV equations (11)-(3) for the background and colliding stars, together with the coordinate transformation (19). These solutions are then conformally transformed and used to compute $\hat{\rho}_{1}$. Finally, Eq. 20) is solved for the perturbation of the conformal factor $\phi_{1}$ as a boundary value problem using a standard tridiagonal solver [30]. The boundary conditions for $\phi_{1}$ in Eq. (20) are: regularity at the axis, $\left.\phi_{1}\right|_{\hat{r}=0}=0$, and asymptotic flatness at infinity, $\left.\frac{d}{d \hat{r}} \phi_{1}\right|_{\hat{r}=\infty}=0$.

The main aim of the present paper was to provide a prescription for close-limit initial data in the case of neutron stars. But, even though we will not discuss a large sample of numerical evolutions here, it is clearly appropriate to illustrate typical results obtained for the proposed neutron star close-limit approximation. We have used two independent numerical codes [19,20] to evolve the relevant perturbation quantities from the initial data obtained in the previous sections. These evolution codes have been well tested, and we have verified that they lead to identical results in the present case.

In Figures if 3 we show a typical initial data and gravitational waves resulting from the close-limit approximation. This particular case pertains to a final stellar configuration with $\rho_{c}^{0}=2.69 \times 10^{15} \mathrm{~g} / \mathrm{cm}^{3}$ and $K_{0}=100 \mathrm{~km}^{2}$. For these parameters, the mass and radius of the background star are $M_{0}=1.24 M_{\odot}$ and $R_{0}=9.0 \mathrm{~km}$, respectively. The initial colliding stars, which are displaced a distance $0.1 R_{0}$ from the center of mass, follow from $\rho_{c}^{*}=2.98 \times 10^{15} \mathrm{~g} / \mathrm{cm}^{3}$ and $K_{*}=90.25 \mathrm{~km}^{2}$ and have $M_{*}=1.17 M_{\odot}$ and $R_{0 *}=8.58 \mathrm{~km}$. Figure 1 ishows the perturbed conformal factor $\phi_{1}$ and density $\rho_{1}$. The character of the emerging gravitational waves is exactly what one would expect: A sharp initial burst followed by slowly damped oscillations. The long-lived oscillations are associated with the various fluid pulsation modes of the final configuration. That this is the case is clear from Figure 3, where we show the Fourier transform of the waveform in Figure 2. In Figures 2 and 3 we can also see that it is rather difficult to draw any conclusions about the relevance of the short-lived $w$-modes from our sample evolution. While the modes are clearly present in the early part of the signal if we graph the variable $S$ as defined by Allen et al [19], they are absent in the corresponding Zerilli function $Z$ which should be a reliable measure of the emerging gravitational waves. However, at the present time this is perhaps neither here nor there: As already mentioned, we expect the assumption of conformally flat initial data to suppress the $w$-modes in a crucial way. Hence, we should not attempt to draw any conclusions about their potential astrophysical relevance from the present results.

We can, however, meaningfully discuss the longer lived fluid modes. The various peaks in the spectrum shown in Figure 3 correspond directly to the fluid pulsation modes of the final configuration, the lowest frequency mode being the fundamental $f$-mode and the next one being the first of the pressure $p$-modes. This is interesting further evidence that these modes will be clearly excited whenever a neutron star is dynamically perturbed, which is highly relevant considering the recently devised method for inferring stellar parameters from detected gravitational waves carrying the mode-signature [17,18]. However, one must still prove that these modes carry sufficient energy to be observable by the new generation of gravitational wave detectors. To investigate this issue, one should study a larger sample of close-limit evolutions and perhaps also attempt a comparison to the black-hole case. Work along these lines is currently in progress, and we hope to report on it soon. 


\section{FINAL COMMENTS}

We have developed a framework for studying merging neutron stars using perturbation theory. Specifically, we have devised suitable initial data for the very late stages of binary merger, when the two merged stars can be considered as a final configuration plus perturbations. This "close-limit" approximation is analogous to the one that has provided surprisingly accurate results in the case of colliding black holes. However, as we have discussed in some detail, it is not straightforward to devise a similar approximation in the case of neutron stars. Our chosen scheme respects some of the required physical constraints. It has the correct limits at infinite and zero separation of the two stars. Furthermore, we have tried to model the changes in the equation of state brought about by the merger in a simple, but seemingly appropriate [6], way. Still, it must be remembered that this is just a first step and that one could potentially refine the close-limit idea considerably.

Even though it is clear that the close-limit approach to neutron star collisions has severe limitations (it will certainly never completely replace fully nonlinear general relativistic hydrodynamics simulations) we think that it can prove to be of considerable use. On a technical level, it should be rather straightforward to use our initial data sets, combined with the perturbation evolutions, as benchmark tests for fully nonlinear evolutions in numerical relativity taking account of the detailed fluid dynamics. This would in fact be a very useful test for a nonlinear simulation since it would ascertain that the detailed dynamics associated with the stars various pulsation modes could be resolved. Also, one could clearly use the perturbation equations to evolve any neutron star process at the late stages, thus saving valuable computing time. Furthermore, it seems likely that we can learn some important physics from our results. Evolutions from all the close-limit data sets that we have so far constructed show that the fluid pulsation modes of the final star are excited to a significant level. As far as the potential excitation of the gravitational $w$-modes are concerned, our present understanding is far from satisfactory, but as we have pointed out, an investigation of this issue likely requires a true understanding of "astrophysical" initial data and a relaxation of the standard assumption of conformal flatness.

In conclusion, it is worth pointing out that our close-limit framework can be extended to more general situations. In the present study we chose to restrict ourselves to the head-on collision of two stars that are initially at rest. These assumptions can conveniently be relaxed to allow the stars to have initial momentum. A generalization to the physically relevant case of slow rotation also seems possible. Work in these directions is in progress.

\section{ACKNOWLEDGMENTS}

This work was partially supported by NATO grants CRG960260 and CRG971092, as well as NSF grants PHY9357219, PHY9407194, PHY9423950, and PHY9800973. JP also acknowledges support from the Pennsylvania State University, the Eberly Family Research Fund at Penn State, and the John S. Guggenheim Foundation.

[1] M. Shibata, M. Sasaki, H. Tagoshi, and T. Tanaka, Phys. Rev. D, 51, 1646 (1995).

[2] R.H. Price and J. Pullin, Phys. Rev. Lett. , 72, 3297 (1994).

[3] P. Anninos, R.H. Price, J. Pullin, E. Seidel and W-M. Suen, Phys. Rev. D, 52, 4462 (1995).

[4] G. Khanna et al, unpublished (1999).

[5] P. Mészáros and M. J. Rees, Astrophys. J., 405, 278 (1993).

[6] S.L. Shapiro, Phys. Rev. D, 58, 103002 (1998).

[7] G.B. Cook, S.L. Shapiro and S.A. Teukolsky, Astrophys. J., 424, 823 (1994).

[8] X. Zhuge, J.M. Centrella and S.L.W. McMillan, Phys. Rev. D, 50, 6247 (1994).

[9] S. Chandrasekhar, Phys. Rev. Lett. , 24, 611 (1970); J.L. Friedman and B.F. Schutz, Astrophys. J., 222, 281 (1978).

[10] N. Andersson, Astrophys. J., 502, 708 (1998).

[11] J.L. Friedman and S. Morsink, Astrophys. J., 502, 714 (1998).

[12] N. Andersson, K.D. Kokkotas and B.F. Schutz, Astrophys. J., 510, 846 (1999).

[13] L. Lindblom, B.J. Owen and S.M.Morsink, Phys. Rev. Lett. , 80, 4843 (1998).

[14] B.J. Owen, L. Lindblom, C. Cutler, B.F. Schutz, A. Vecchio and N. Andersson, Phys. Rev. D, 58, 084020 (1998).

[15] P.R. Brady and T. Creighton, gr-qc/9812014.

[16] N. Andersson and K.D. Kokkotas, Phys. Rev. Lett. , 77, 4134 (1996).

[17] N. Andersson and K.D. Kokkotas, Mon. Not. R. astr. Soc., 299, 1059 (1998). 
[18] K. D. Kokkotas, T. A. Apostolatos, N. Andersson, gr-qc/9901072.

[19] G.D. Allen, N. Andersson, K.D. Kokkotas and B.F. Schutz, Phys. Rev. D, 58, 124012 (1998).

[20] J. Ruoff, P. Laguna and J. Pullin, in preparation (1999).

[21] F.J. Zerilli, Phys. Rev. D, 2, 2141 (1970).

[22] W. Krivan, P. Laguna, P. Papadopoulos and N. Andersson, Phys. Rev. D, 56, 3395 (1997).

[23] K.D. Kokkotas and B.F. Schutz, Mon. Not. R. astr. Soc., 255, 119 (1992).

[24] R. Arnowitt, S. Deser and C.W. Misner: in Gravitation ed. L. Witten (Wiley, New York, 1962) p. 227.

[25] J.W. York, Jr., in Sources of Gravitational Radiation, ed. L. Smarr (Cambridge University Press, Cambridge, England, 1979), p. 83.

[26] K. Camarda, P. Laguna and D. Arnold, in preparation (1999).

[27] N. Andersson, K.D. Kokkotas, P. Laguna and P. Papadopoulos, in preparation (1999).

[28] J.M. Bowen, Gen. Rel. Grav., 11, 227 (1979); Gen. Rel. Grav., 14, 1183 (1982); J.M. Bowen and J.W. York, Phys. Rev. D, 21, 2047 (1983).

[29] F.A. Rasio and S.L. Shapiro, Astrophys. J., 432, 242 (1994).

[30] W.H. Press, S.A. Teukolsky, S.A. Veteerling and B.P. Flannery, Numerical Recipes (Cambridge University Press, Cambridge, England, 1992). 

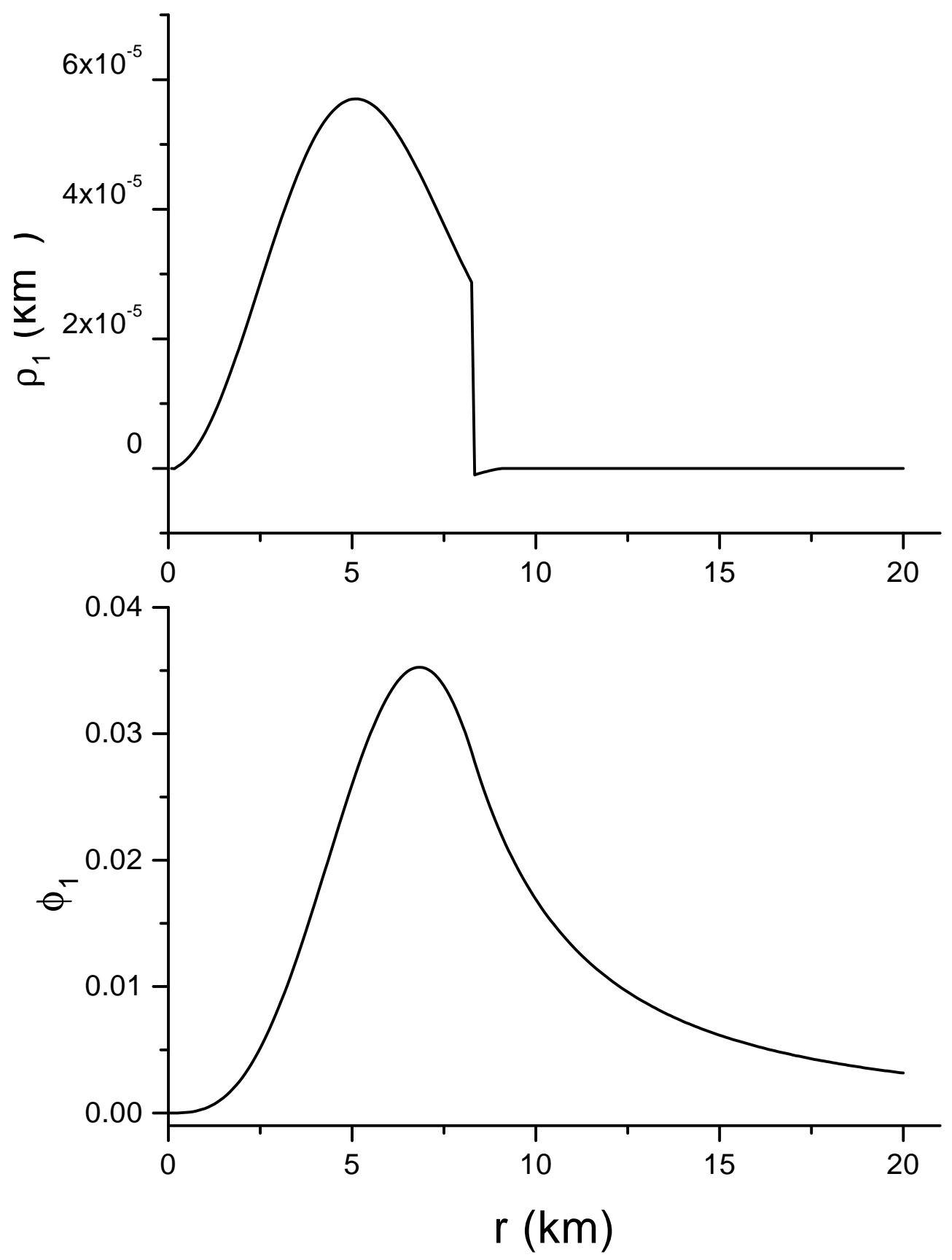

FIG. 1. Perturbations of the conformal factor $\phi_{1}$ and density $\rho_{1}$ for a close-limit collision of equal-mass stars with conformal separation $\xi=0.1 R_{0}$. The TOV parameters for the background and colliding stars are: $\rho_{c}^{0}=2.69 \times 10^{15} \mathrm{~g} / \mathrm{cm}^{3}$ and $K_{0}=100 \mathrm{~km}^{2}$. For these parameters, the mass and radius of the background star are $M_{0}=1.24 M_{\odot}$ and $R_{0}=9.0 \mathrm{~km}$, respectively. The initial colliding stars, which are displaced a distance $0.1 R_{0}$ from the center of mass, follow from $\rho_{c}^{*}=2.98 \times 10^{15} \mathrm{~g} / \mathrm{cm}^{3}$ and $K_{*}=90.25 \mathrm{~km}^{2}$ and have $M_{*}=1.17 M_{\odot}$ and $R_{0} *=8.58 \mathrm{~km}$. 


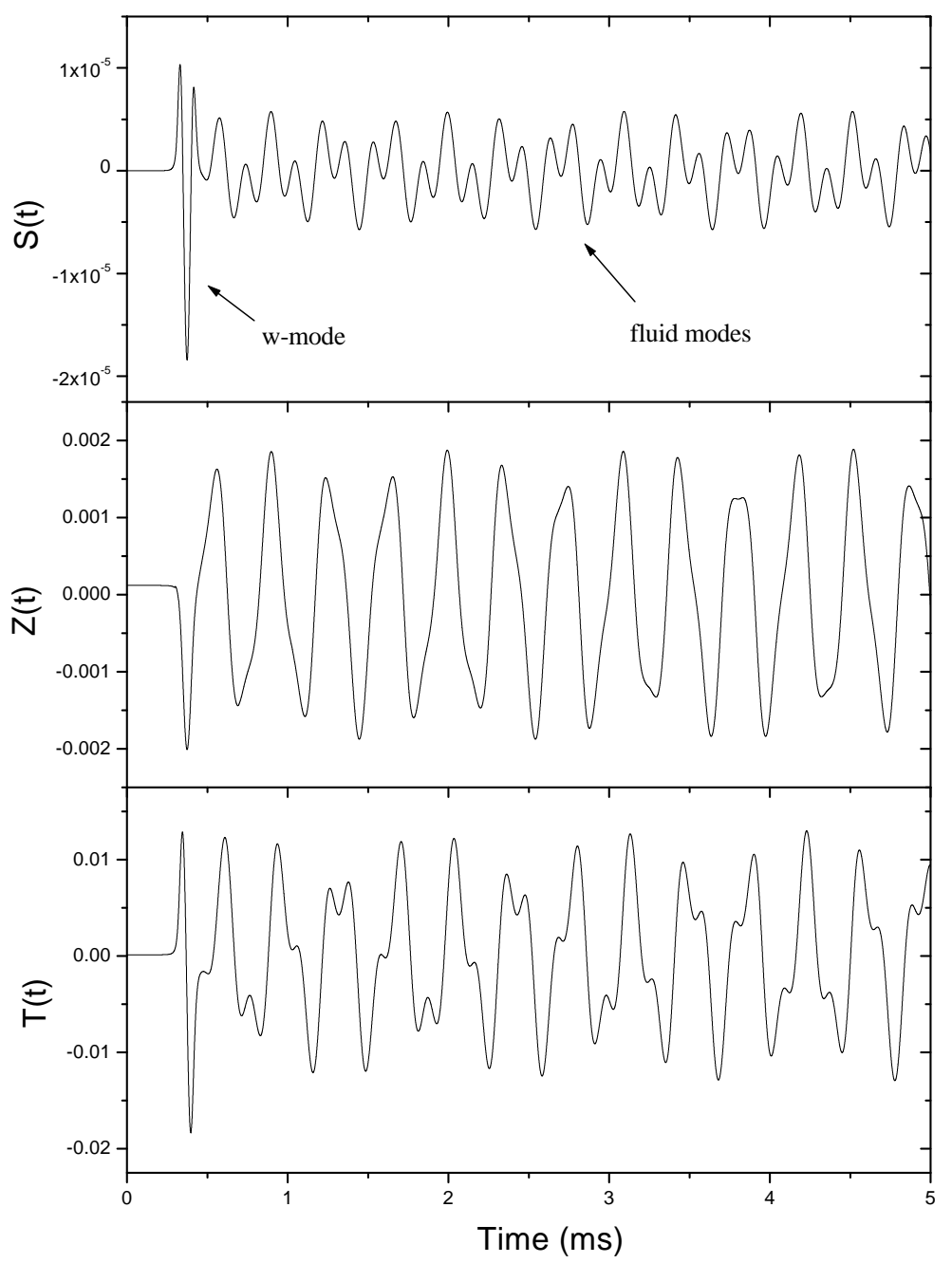

FIG. 2. Snapshots of various functions illustrating for the evolution of the initial data shown in Fig. 1. We show the function $S$ defined as in [19], the Zerilli function and the perturbed conformal factor $T=4 r \phi_{1} / \phi_{0}$. 


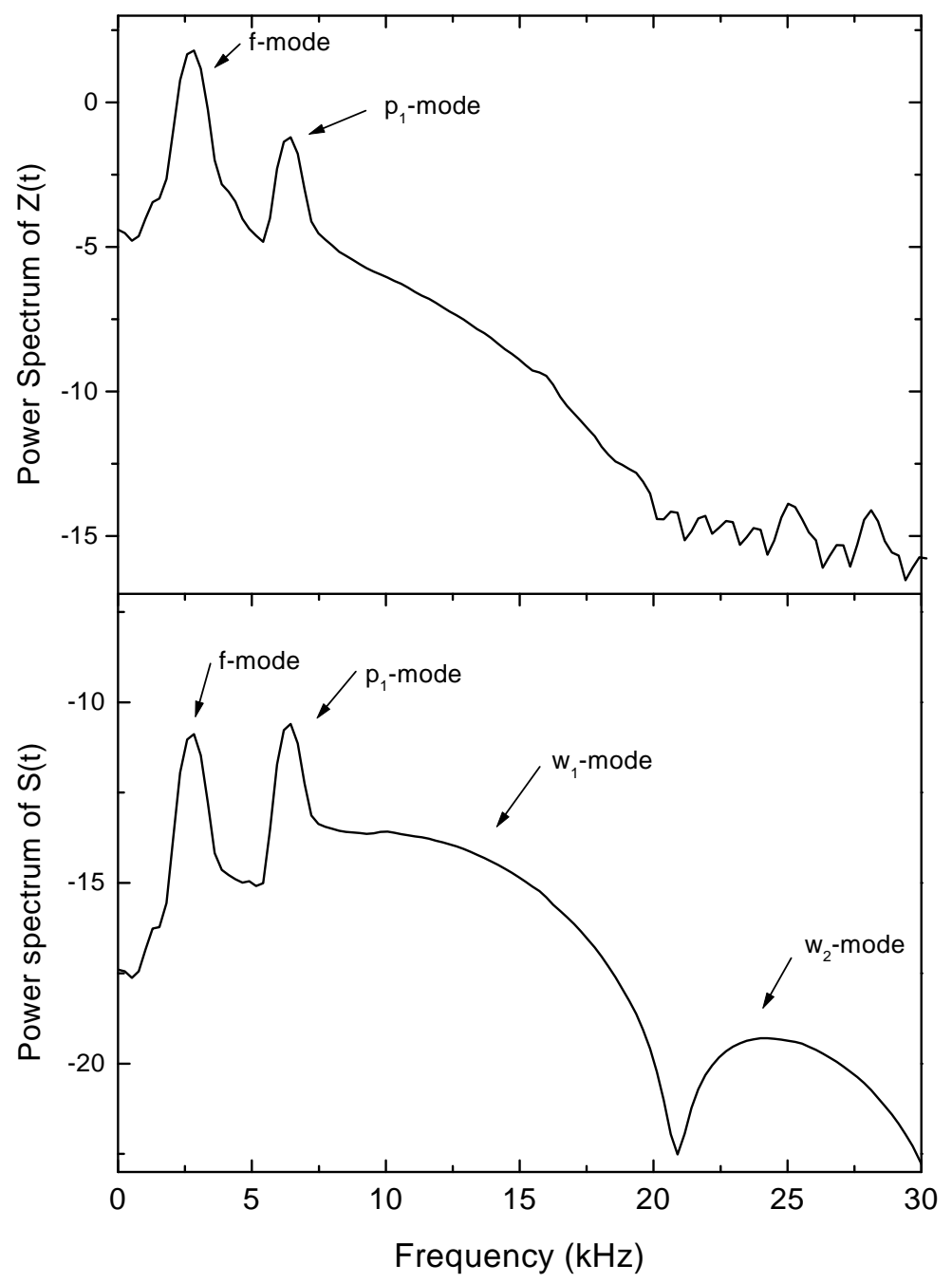

FIG. 3. The Fourier transform of the waveforms for the function $S$ and the Zerilli function $Z$ shown in Fig. 2. The two sharp peaks correspond to the lowest frequency fluid pulsation modes of the final configuration. The first peak belongs to the fundamental $f$-mode while the next one is the first of the pressure $p$-modes. It is notable that $w$-modes seem to be present in $S$ but not in $Z$. This effect is further discussed in the main text. 